\title{
Enhancement of Poly-crystalline Silicon Solar Cells using Nanotechnology
}

\author{
Darshan Bhavesh Mehta \\ Manipal Institute of Technology, \\ Manipal, India 576104
}

\begin{abstract}
The solar cell industry has developed radically in recent years due to strong interest in renewable energy and the problem of global climate change. Cost is a primary concern in the success of any solar technology. The solar cells nowadays are simply not efficient enough and are too costly to manufacture for large-scale electricity production. Nevertheless, potential advancements in Nanotechnology may change this. Use of Nanotechnology will make the production of solar cells cheaper and also more efficient. Nanotechnology has shown huge breakthroughs in the solar field. Quantum dots have the capacity to change the world. They are a type of solar cells which are entirely beyond something you might imagine. Nanotechnology may be able to slightly increase the efficiency of solar cells, but the most capable merit of Nanotechnology is the decrease of manufacturing cost. Photo voltaic based on Cadmium telluride, Copper indium gallium selenide (CIGS), copper indium selenide (CIS), and organic substances are being made with the aim of decreasing the cost per Watt even if that means giving up its conversion efficiency and dependability. Utilizing nanotechnology in solar cell would be cheaper alternative to the conventional solar cells.
\end{abstract}

\section{Keywords}

Nanotechnology, Quantum dots, Photo-voltaic cells, Solar PV cell.

\section{INTRODUCTION}

Conventional solar cells have two main shortcomings: efficiencies and their expensive manufacturing cost. The first shortcoming, inefficiency, is almost inevitable with silicon cells. This is for the reason that the incoming photons, or light, must have the correct energy, called the band gap energy, to knock out an electron.

If,

Energy of photon < band gap energy, then it will pass through

Energy of photon > band gap energy, then that extra energy is lost heat.

These two effects solely result in loss of around 70 percent of the radiation energy that is incident on the cell.

Nano particles are specks of matter smaller than 1/10000 times than the width of a human hair. And since they are small, a large part of these particles` atoms lie on their surfaces rather than in their centers. Therefore the surface interactions dominate Nano-particle behavior. And therefore most of the times they have dissimilar Due to multiple reflections, the effective optical path for absorption is greater than the actual film thickness.
1. Light generated electrons and holes need to travel over a much smaller path and therefore losses due to recombination are dramatically decreased.

2. Therefore, the absorber layer thickness in Nanostructured solar cells can be as small as $150 \mathrm{~nm}$ instead of several micrometers in the conventionally thin film solar cells.

3. The energy band gap of several layers can be made to the desired design value by varying the size of Nano-particles. This permits for more design flexibility in the absorber of solar cells.

Thin film is a more cost-effective solution and uses a lowpriced support onto which the active component is applied as a thin layer. By this method material used is reduced to about $1 \%$ as compared with wafers and costs are reduced too. Many of these cells use amorphous silicon which doesn't have a crystalline structure and subsequently has a much lower efficiency of about $8 \%$, though it fabrication is very economical. Comparison of different photo-voltaic cell shown in table.

\section{Visible Light Spectrum}

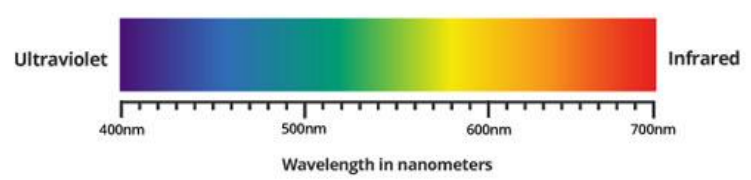

Figure 1. Visible Light Spectrum

\section{NANOTECHNOLOGY BOOSTS SOLAR CELLS PERFORMANCE}

Present day solar cells are unable to convert the entire energy of the incoming light into usable energy because some of the light can escape back out of the cell into the atmosphere. Furthermore, the light from the sun is made up of several colors and the cell may be more efficient at converting blue light while being less efficient at converting red light. See in Figure 1. Lower energy light passes through the cell unexploited. Higher energy light does excite electrons to the conduction band, but any energy beyond the band gap energy is lost as heat. If these excited electrons spontaneously recombine with the created holes not if they are not captured and redirected and the energy will be lost as heat or light.

In traditional solar cells, UV light is filtered out or absorbed by the silicon and transformed into potentially damaging heat and not electricity. UV light can efficiently couple to correctly sized Nano-particles and generate electricity. Integrating a high-quality film of silicon Nanoparticles, $1 \mathrm{~nm}$ in size 
directly onto $\mathrm{Si}$ solar cells improves the power output by about $60 \%$ in the UV range of the light spectrum. In bulk material, the radius is much smaller than the semiconductor crystal. The "continuous band" of electron energy intensities no longer can be regarded as continuous since Nano-crystal diameters are smaller than the Bohr radius. The energy levels become distinct, and quantum confinement is seen to operate. The variance of a few atoms between two quantum dots alters the band gap boundaries. Small Nano-crystals absorb shorter wavelengths or bluish light, whereas larger Nano-crystals absorb longer wavelengths or reddish light. The band gap energy level changes on altering the shape of the dot.

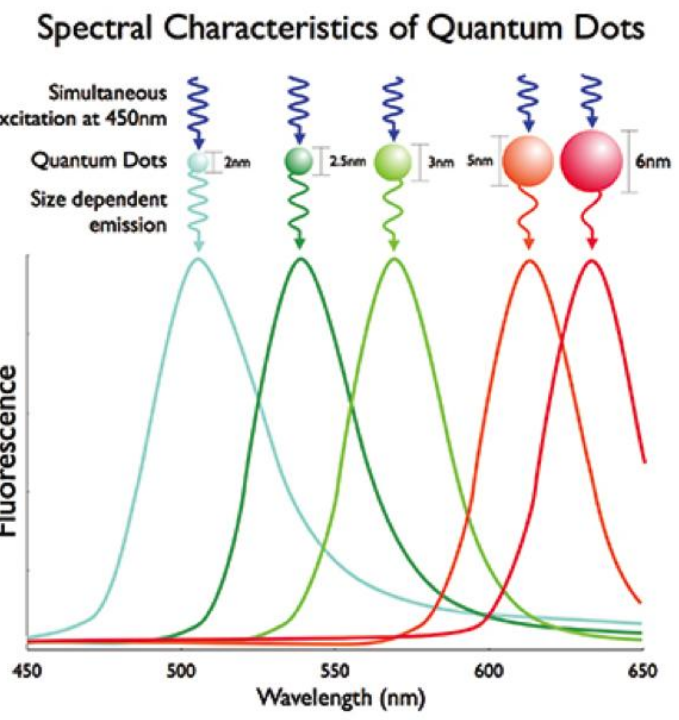

Figure 2. The relationship of size of quantum dot to the light absorbed

To develop the enhanced solar cells, the researchers began by first changing bulk silicon into discrete, Nano-sized particles. Conditional on their size, the Nanoparticles will fluoresce in different colors. Nanoparticles of the favored size were then isolated in isopropyl alcohol and distributed onto the face of the solar cell. Since alcohol evaporated, a film of tightly crammed Nanoparticles was left firmly fastened to the solar cell. Solar cells layered with a film of $1 \mathrm{~nm}$, blue luminescent particles showed a power optimization of $60 \%$ in the UV range of the light spectrum, but less than $3 \%$ in the visible light range. Solar cells coated with $2.85 \mathrm{~nm}$, red particles showed an improvement of $67 \%$ in the UV range, and about $10 \%$ in the visible range of the light spectrum. Extremely thin films of vastly mono-dispersed luminescent Silicon Nanoparticles are directly integrated on polycrystalline Silicon solar cells. Films of 1 Nanometer blue luminescent or 2.85 Nanometers red luminescent Silicon Nano-particles generate high voltage improvements with improved power output of $60 \%$ in the UV-blue range. In the visible, the improvements are of approximately $10 \%$ for the red and about $3 \%$ for the blue particles.

An additional key feature of these solar cells is that the Nano-rods possibly can be 'tuned' to absorb different wavelengths of light which can considerably improve the efficiency of the solar cell since additional of the incident light can be exploited. Single-walled CN T to a film developed of $\mathrm{TiO}_{2}$ Nano-particles, increasing the efficiency of converting ultraviolet light into electrons to almost twice as much as when compared with the performance of the Nanoparticles alone.

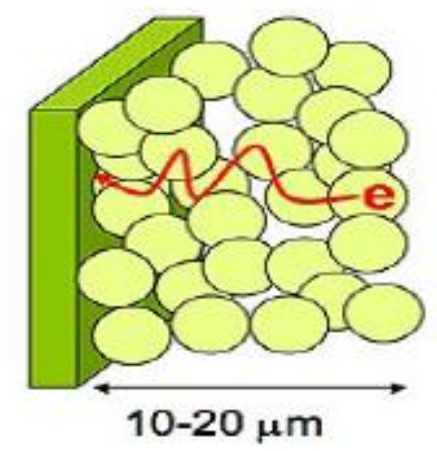

Figure 3. Escape route of electron

Escape route: Electrons generated in a Nano-particle-based solar cell have to shadow a circuitous path (red line) to reach an electrode. Most electrons do not make it, decreasing the efficiency of these cells. Scientists at Notre Dame have used carbon Nano-tubes to aid the electrons reach the electrode and thereby improving their efficiency. Short of the CNT, the electrons produced when the light is absorbed by $\mathrm{TiO}_{2}$ particles have to bounce from particle to particle to reach an electrode. Most of them never make it out to produce current. The carbon Nano-tubes "collect" the electrons and offer a more direct route to the electrode, improving the efficiency of the solar cells.

A much better electron ballistic transport property is offered by carbon nano-tubes along its axis with large current density capacity on the surface of the solar cell without much loss. A very high efficiency in photovoltaic conversion is due to the alignment of the carbon nano-tubes with the polymer composites substrate. As there is an increases in contact area of the polymer, the better charge transfers and energy conversion takes place in the composites. In this system, the efficiency of solar cell is approximately $50 \%$ at the laboratory scale. The optimum efficiency is achieved with the use of an aligned carbon nano-tube with photo-voltaic cell. P3OT has been successful in improving the efficiency due to the presence of the polymer and Nano-tubes junctions within the polymer matrix. The high electric field created inside the Nano tube splits the energy in a crystal to an excited electron and an associated hole and enables faster electron transfer also improves the quantum efficiency by $50 \%$.

\subsection{Improving the Efficiency of Solar Cells by Using Semiconductor Quantum Dots (QD)}

Another approach to increase the conversion efficiency of solar cells is the use of a semiconductor crystal of Nanometer dimensions with distinctive conductive properties determined by its size also known as the quantum dots (QD). With the usage of the quantum dots, the band gaps can be adjusted specifically to convert light of larger wavelength and thus increase the efficiency of the solar cells. The quantum Dots Solar Cells are contrived using, III/V-semiconductors and other alloys such as combinations of Silicon and Germanium or Silicone and Beryllium or Titanium and Selenium can be considered as well. Few potential advantages of the Silicone and Germanium quantum Dot solar cells are that they provide higher light absorption in particular in the infrared 
spectral region, compatibility, increase of the photo current at higher temperatures, improved radiation hardness compared to conventional solar cells.

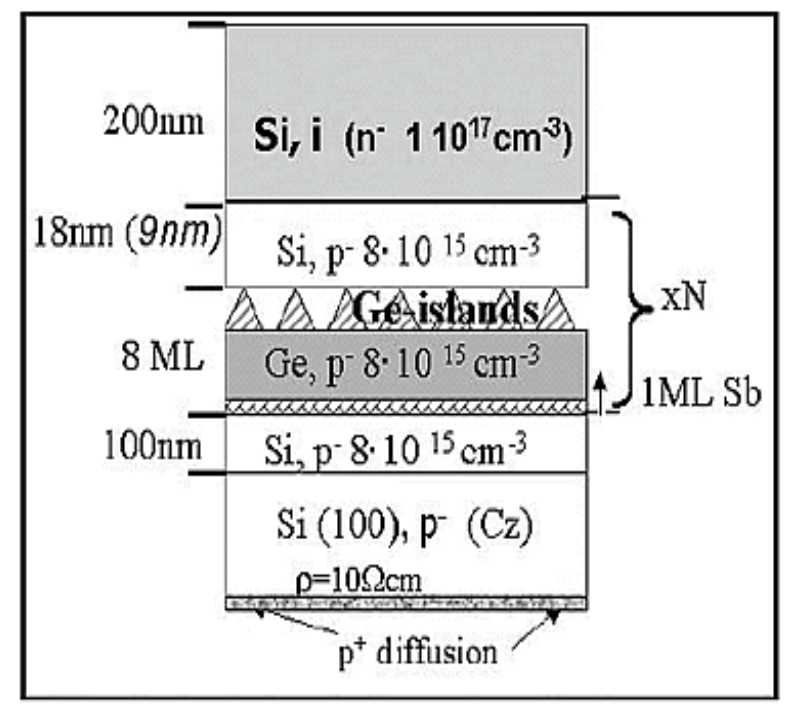

Figure 4. Schematic structure of a $\mathrm{Si} / \mathrm{Ge} \mathrm{QD}$ solar cell with layers Ge quantum dots in the active layer of the Si solar cell substrate.

\section{NANOTECHNOLOGY IMPROVE THE SOLAR CELL}

Presently available Nanotechnology adopted solar cells are not as efficient as the conventional ones, however their lower cost counterbalances this. Perceiving the future of Nanotechnology, the quantum dot solar cells should both be cheaper and, be able to reach higher efficiency levels than conventional ones. The Nanoparticle along with quantum dots are coated with tiny semiconductor crystals.

Advantage of using quantum dots is that it has the potential to convert high-energy photons into multiple electrons. Like the conventional system the quantum dots, for every photon of sunlight that hits the dots it produces three electrons. These electrons move from the valance band into the conduction band. The dots increase conversion efficiency to as high as 65 percent by catching more spectrum of the sunlight waves.

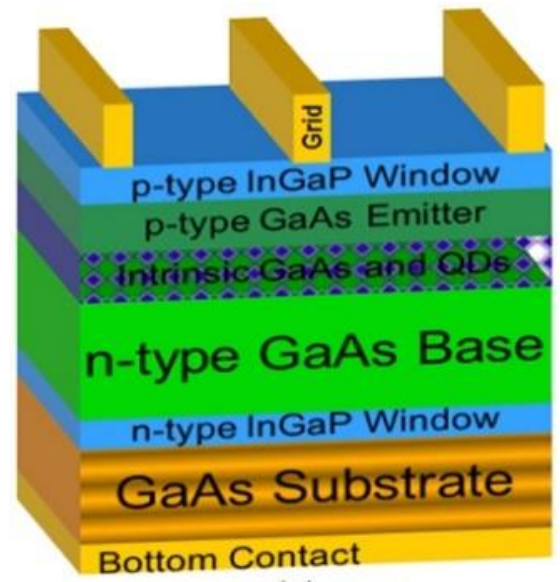

Figure 5. Quantum-dot enhanced solar-cell design concept

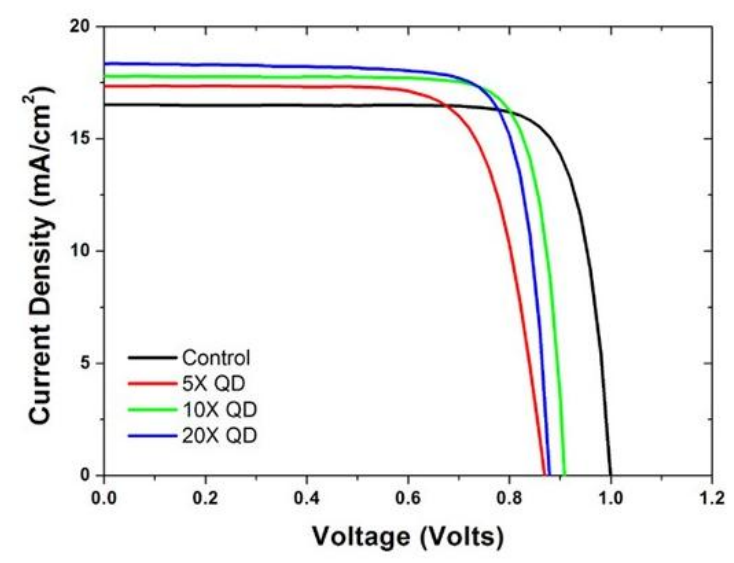

Figure 6: Current density voltage curves for control and 5-20 layer enhanced cells under one sun global air mass 1.5 (AM1.5g) light. These cells did not have anti-reflective

Another usage of the quantum dots is that it could be used is in making so-called hot carrier cells. Classically the extra energy supplied by a photon is lost as heat, whereas using a hot carrier cell, the extra energy from the photons result in higher-energy electrons which in turn lead to a higher voltage.

The key issue in achieving higher photo conversion efficiency is the movement of electrons across the particle network is. Application of network made of Carbon NanoTubes as support to anchor light ingathering semiconductor particles by assisting the electron passage to the collecting electrode surface in DSSC. Charge injection from excited CdS into SWCNT excitation of CdS Nanoparticle. When Carbon Nano-tubes attached in CdSe \& CdTe induces charge transfer under visible light irradiation, the enhanced interconnectivity between the $\mathrm{TiO}_{2}$ particles and the MWCNTs in the porous $\mathrm{TiO}_{2}$ film was found to be the reason of the enhancement in short circuit current density.

\section{COST REDUCTION BY NANOTECHNOLOGY}

A process similar to printing which uses the low temperature to manufacture conventional crystalline silicon solar cell. Nanotechnology reduced installation costs. This was achieved by producing flexible temperature rolls for vacuum deposition but at the same time it reduced manufacturing costs. Cells made from semiconductor thin films will also have this characteristic. One of the companies have successfully created a solar coating that is the most cost efficient solar energy source ever made. Their solar sheet cells differ from the current solar technology systems because of reduced the cost of production from $\$ 3$ a watt to a mere 30 cents per watt which makes it cheaper than charcoal.

As a matter of fact, the photovoltaic devices are limited to lower efficiencies practically which is governed by the thermodynamic limits and production costs that involve negotiations in materials, production processes, and photovoltaic device packaging. The Lewis Group as a result of higher efficiency or lower production provides a thorough illustration of the efficiency trends for various photo-voltaic devices materials such as crystalline silicon used in semiconductors as well as the new approaches to thin film photo-voltaic including amorphous silicon, cadmium telluride (CdTe), copper indium deselenide (CIS) and copper indium 
gallium deselenide materials (CIGS). These thin film material could offer considerable price reductions costs.

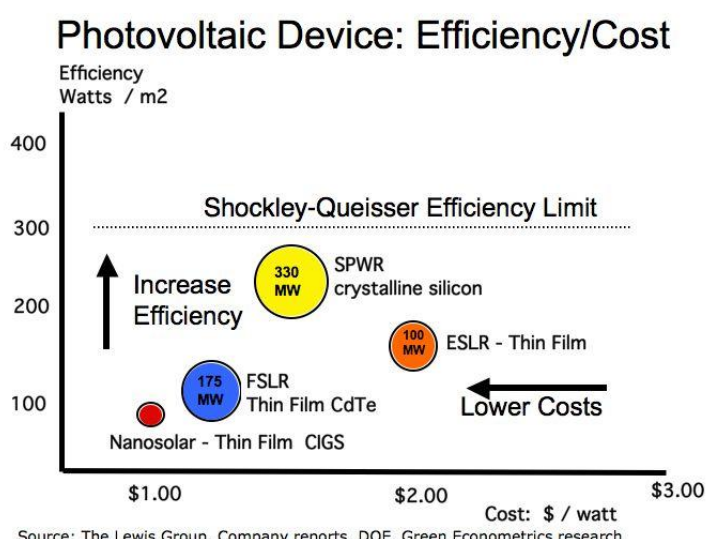

Figure 7. Cost/Efficiency Tradeoff.

\section{CONCLUSION}

1. Coating existing roofing materials with its plastic photovoltaic cells which are inexpensive enough to cover a home's entire roof with solar cells and then enough energy could be captured to power almost the entire house.

2. If many houses did this then our dependence on the electric grid (fossil fuels) would decrease and help to reduce pollution.

3. Preservation of non-renewable sources of energy by usage of inexpensive solar cells. These cells when installed on the roof of a house have the capacity to power the entire house with one day of charge.

4. These solar cells have applications across the military as well. The U.S. Army has hired Konarka Technologies to help design a better way to power their soldiers' electrical devices. According to Daniel McGahn, Konarka's executive vice president, "A regular field soldier carried 1.5 pounds of batteries those days before the renewable sources came into picture. Since these renewable sources have a longer time out, they only have to carry 60 to 70 pounds inexpensive and reasonably efficient solar equipment soldier." Still if Nanotechnology can be used to manufacture solar cells, it would greatly improve a soldiers' mobility.

5. Inexpensive solar cells would also help provide electricity for rural areas or third world countries. Since the electricity demand in those areas is lower hence making solar cells the ideal source of energy.
6. Solar water heating, solar cookers, solar powered medical equipment, saves money by reducing the electricity bills and helps millions of people in electric grid crisis. Also invention of solar electric vehicles has contributed in saving fossil fuels to a greater limit.

The unification of Nanotechnology and solar energy promises enhancement of efficiency of the solar cells at the same time reducing the manufacturing and maintenance cost

\section{REFERENCES}

[1] Nayfeh, "Thin film silicon Nanoparticle UV photodetector" IEEE Photonics Technology, Volume 16, Issue 8, Pages 1927-1929, August 2004.

[2] Nayfeh, "Enhancement of polycrystalline silicon solar cells using ultrathin films of silicon Nanoparticle" Applied Physics, Volume 91, Issue 6, Article 063107, August 6, 2007.

[3] Aldous, Scott. "How Solar Cells Work." How Stuff Works. 22 May 2005. <http://science.howstuffworks.com/solar-cell1.htm>.

[4] K.R. Catchpole and A. Polman "Plasmonic Solar Cells,", Optics Express, Vol. 16, Issue 6, December 22, 2008, Focus Issue on Solar Energy edited by Alan Kost, University of Arizona.

[5] M. Topinka 'Carbon Nanotube electrodes for solar cells'. SPRC Workshop: Solar Cell Technology Options: Paths to Lower \$ per Watt, Stanford University, Stanford, Calif. Feb. 24, 2007.

[6] M.W. Rowell (June 5, 2006). 'Organic solar cells with carbon Nanotube network electrodes'. APPL PHYS LETT, 233506.

[7] B.J.Landi, R.P. Raffaelle , S.L.Castro , S.G.Bailey ,C.M.Evans (May2005) 'CdSe quantum dot single Wall carbon Nanotube complexes for polymeric.

[8] B.J.Landi , R.P. Raffaelle , S.L.Castro , S.G.Bailey (March 2005) 'Single Wall carbon Nanotube-polymer solar cell' . Progree in photovoltics: Research \& Applications 13 (2): 165-17.

[9] Greg P. Smestad," The Basic Economics of Photovoltaics" Optical Society of America 2008.

[10] Harin S. Ullal," Polycrystalline Thin-Film Photovoltaic Technologies: Progress And Technical Issues" $19^{\text {th }}$ European PV Solar Energy Conference and Exhibition Paris , France June 7-11, 2004.

[11] Claudio Pelosi and Matteo Bosi." Light concentration increases solar-cell efficiency and reduces cost ",17 May 2007, SPIE Newsroom. DOI: 10.1117/2.1200704.0689. 